\title{
Study Effect of Objects Orientation in the Mediums On GPR Signal Reflections Using FDTD Simulation
}

\author{
Aye Mint Mohamed Mostapha, Gamil Alsharahi, and Abdellah Driouach
}

\begin{abstract}
Ground penetrating radar (GPR) is a very effective tool for detecting and identifying objects below the ground surface. based on the propagation and reflection of high-frequency electromagnetic waves. The GPR reflection can be affected by many things like the type of objects orientation, their shapes etc. The purpose of this paper is to study by simulation the effect of objects orientation in two different mediums (dry and wet sand) on the GPR signal reflection using Reflexw software which is based on a numerical method known as finite difference in time domain (FDTD).The simulations that have been realized included a conductor and dielectric objects. The results obtained have led us to find that the propagation path, the reflection strength and the signal form change with the change of object orientation and nature. To confirm the validity of the results, we compared them with experimental results previously published by researchers under the same conditions.
\end{abstract}

Index Terms-GPR; Simulation; Orientation; FDTD; Reflection.

\section{INTRODUCTION}

Ground penetrating radar (GPR) is a geophysical inspection tool which uses electromagnetic (EM) waves, typically in the frequency range $10-3000 \mathrm{MHz}$, to image structures and features buried in the ground [1]-[2]. It can be used in different areas of research such as in planetary sciences civil; environmental engineering; geology and archaeology etc. [3].The major advantages of GPR are the ability to perform scans in a continuously over a large area in a relatively short time. In the additional GPR data can be viewed in real time, allowing the quality of the data acquired directly in the field to be evaluated and possibly the parameters and acquisition parameters to be adjusted [4]. The GPR waves can penetrate tens to hundreds of meters below the lunar surface depending on the frequency of the antenna uses and conductivity of medium, and therefore the received echo contains rich information about near surface stratigraphic structure [5]. The polarization of the electromagnetic wave is a fundamental property of propagation that offers the GPR a unique opportunity to produce enhanced object images in the subsurface. Thenature of the antenna-target polarization and target size play also an important role in obtaining a precise GPR signal. This signal has a significant sensitivity in the different polarization mode [6]. this work presents a simulation study of the effect of objects polarization on the GPR signal reflection using the finite-difference time domain (FDTD) which is considered as one of the effective and accurate numerical simulation technique for electromagnetic wave propagation.

\section{PRINCIPLE OPERATION OF GPR}

A GPR system consists of two antennas, one for the emission of electromagnetic waves and the other for the reception of these same waves, in addition the control unit. During a GPR analysis, The GPR antenna emits a short pulse in the direction of the ground. The generated electromagnetic wave propagates in the medium.

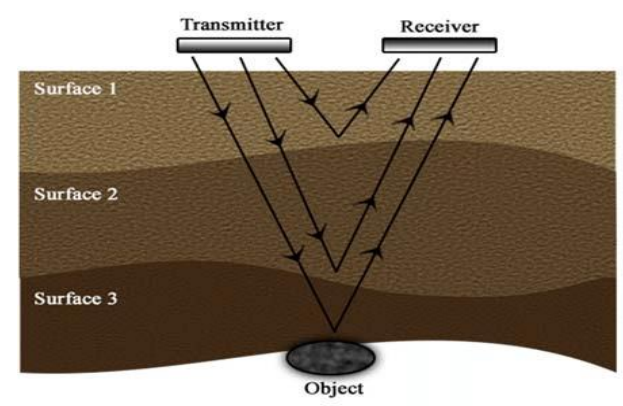

Fig.1. Show pattern principal operation of GPR

If an obstacle is encountered, a reflected wave returns to the ground surface and is detected by the receiving antenna [7],[8], and converted into a new pulse which is amplified, sampled and transmitted to the control unit for processing and recording as a function of time. at each position of the antenna a recording pulse as a function of time is performed. Each record constitutes a trace. The juxtaposition of these traces next to each other constitutes a hyperbola. The apex of this hyperbola corresponds to the position where the middle of the two antennas is just above the object. The further away from the position mentioned previously, the more the travelling time increases the two branches of the hyperbola are obtained as shown in Fig. 1. [9]-[10].

\section{RESULTS AND DISCUSSION}

In this work we simulate the detection of two types of objects with the same dimensional $(0.5 \mathrm{X} 0.05 \mathrm{~m})$ buried with different types of orientation in a medium of dry sand characterized by $0.001 \mathrm{~ms} / \mathrm{m}$ in conductivity and 6 units in relative permittivity and other of wet sand characterized by $0.1 \mathrm{~ms} / \mathrm{m}$ in conductivity and 20 units in relative permittivity. The different simulations are in $2 \mathrm{D}$, and the frequency of the antenna used is $800 \mathrm{MHz}$. 


\section{A. Three conductor objects buried horizontally}

Fig. 2. a presents a model of three conductor objects of the same dimensions buried with different orientation in medium (dimensions $6 \mathrm{~m} \times 2 \mathrm{~m}$ ) of dry sand at a depth of $1 \mathrm{~m}$ and (Fig. 3. a) presents the same objects buried in a medium of wet sand.

Fig.2. b and Fig. 2. c show the radargram obtained and the evolution of the wave radar over time through these figures we can see how the shape of the reflected wave from object changes with the change of its orientation, which produce hyperbolas with different shapes indicates the existence of these objects the presence of the first object (at an angle of 0 ) is indicated by the appearance of two hyperbolas in white and black color, however, we see that for an angle of 45 the object is presented by several hyperbolas corresponding to several consecutive reflections of signal some of these hyperbolas are almost invisible cause of the decrease in reflected signal amplitude from this object at certain times. whereas for the latter case (an angle of 90) the object is represented by three hyperbolas due to three different reflections (Fig. 2. c).

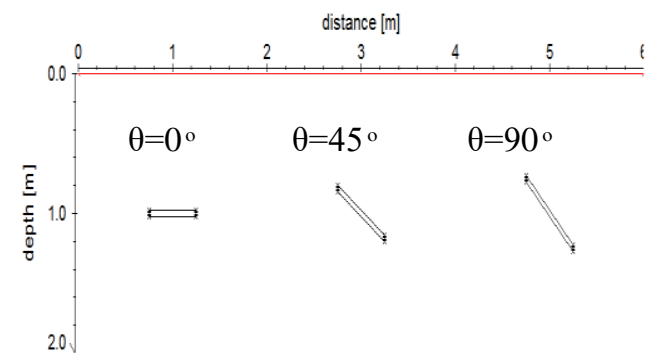

(a)
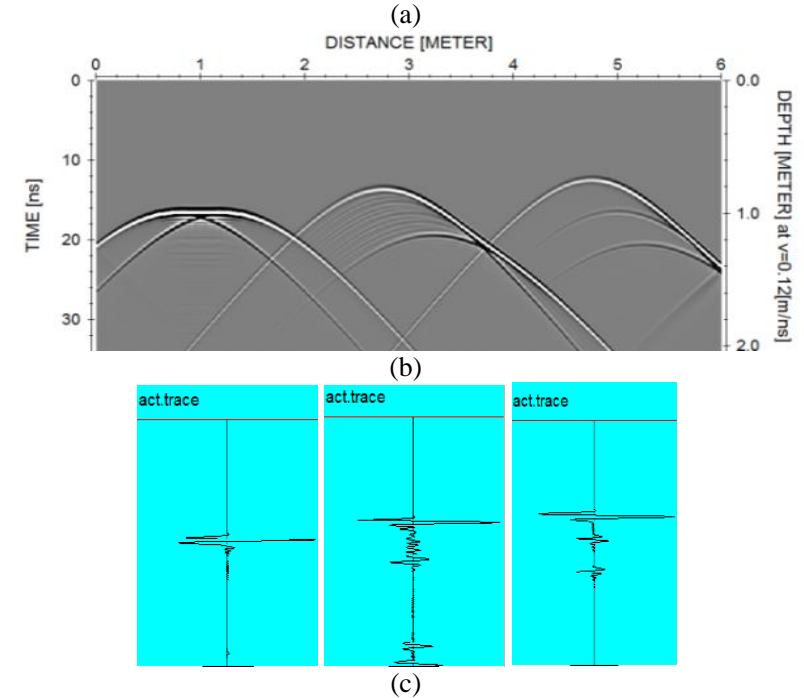

Fig.2. (a) Geometric of three conductor objects buried in a medium of dry sand $\theta$ (angle between antenna and object) $=0,45$ and $90^{\circ}$, (b) Radargram of detection objects and (c) evolution of the wave over time.

The results obtained in the wet sand medium (Fig.3. a and b) show change in the shape of reflected waves from objects and that the hyperbolas are less clear compared to the case of dry sand due to the high permittivity of this medium which diminishes the reflection coefficient of waves.

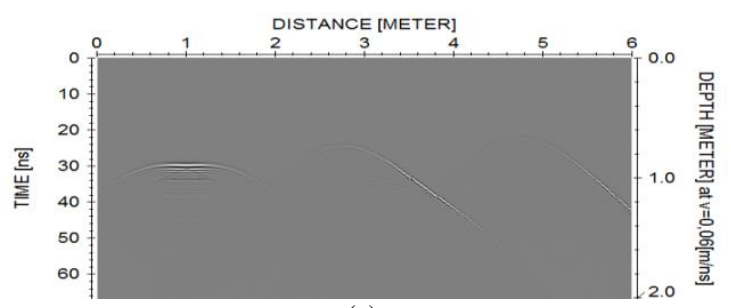

(a)

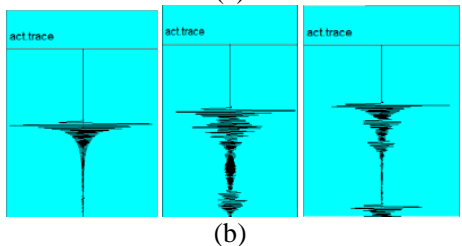

Fig.3. (a) Radargram of detection three conductor objects buried in a medium of wet sand $\theta$ (angle between antenna and target) $=0,45$ and 90; (b) evolution of the wave over time.

\section{B. Three conductor objects buried vertically}

The objects now are placed vertically we note difference in the shape of hyperbolas and waves propagation compared to the previous case and we can see that the hyperbols looks less clear due to the reduction in the amount of reflected energy from objects in this situation.
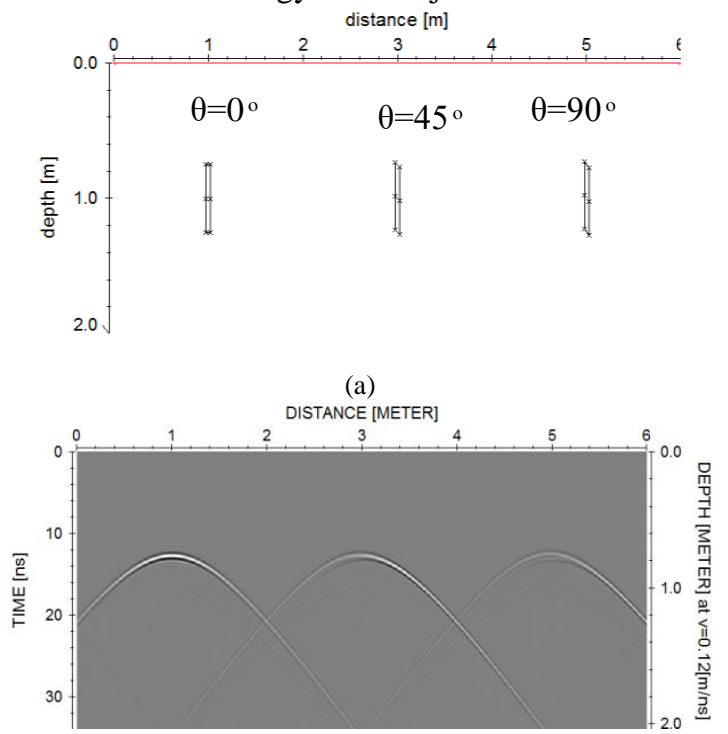

(b)

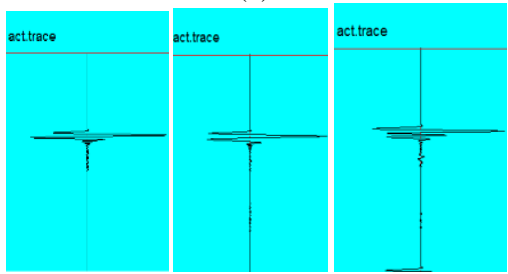

(c)

Fig.4. (a) Geometric of three conductor objects buried vertically in a medium of dry sand with $\theta$ (angle between antenna and target) $=0,45$ and $90^{\circ}$, (b) Radargram of detection objects and (c) evolution of the wave over time.

As for the wet sand the objects are almost invisible (Fig. 5. a).

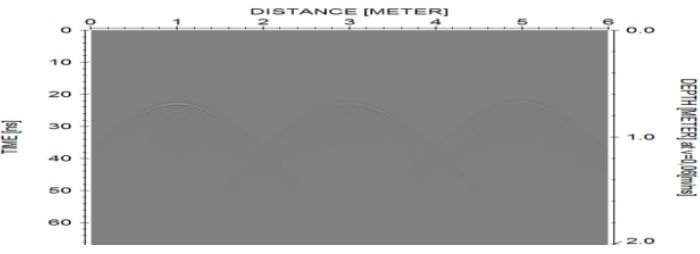


(a)

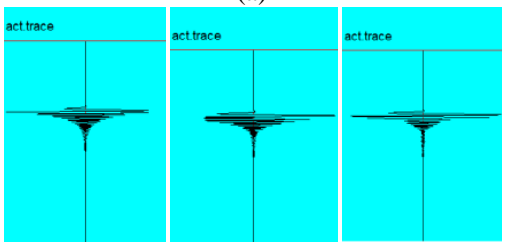

(b)

Fig.5. (a) Radargram of detection of three conductor objects buried vertically in a medium of dry sand with $\theta$ (angle between antenna and target) $=0,45$ and $90^{\circ}$, (b) evolution of the wave over time.

\section{Two objects buried vertically one under the other}

Two cases were studied of two objects dielectric and conductor placed vertically one under the other with two different types of orientation $(0,45)$. In a first case, the dielectric object is placed above the conductor object in a second case we exchange the positions of the two objects (the conductor object is now placed above the dielectric object Fig.7a).

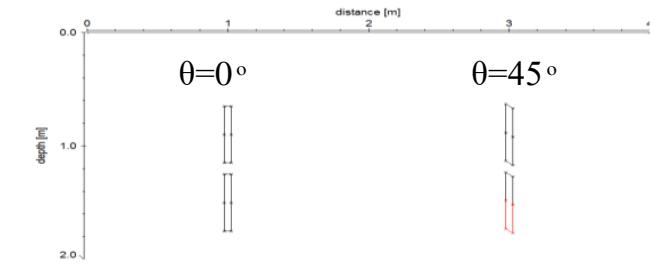

(a)

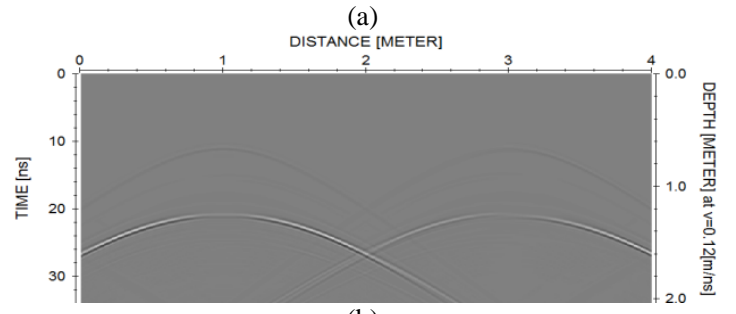

(b)

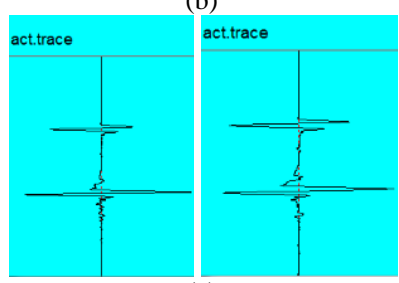

(c)

Fig.6. (a) Dielectric object placed above conductor object $\theta=0,45$; (b)radargram of detection objects; (c) evolution of the wave over time.

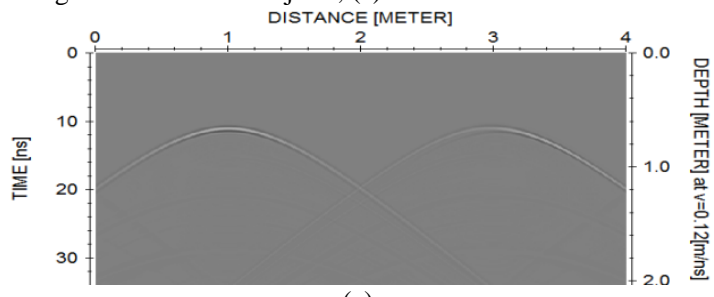

(a)

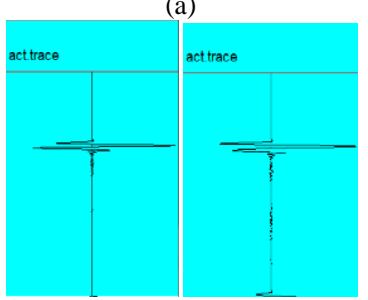

(b)

Fig.7. (a) Radargram of detection conductor object placed above dielectric object $\theta=0,45$;(b) evolution of the wave over time.
For the first case (figure 6c) we can observe that the reflections of conductor objects at both types of orientation (angle 0 and 45) are stronger than the reflection of dielectric objects due to the high electrical conductivity of these objects for this reason the hyperbolas indicate the presence of these objects looks clearer than the others which show the presence of dielectric objects (figure 6b). But for the second case we see only the hyperboles which indicates the existence of the conductor object (figure 7a) this is a result of their higher electrical conductivity they, are considered as good signal reflectors which diminishes the energy of the signal that reaches the dielectric objects. For the wet sand medium, we notice the attenuation of the wave (figure $8 \mathrm{~b}$ ) and the non-detection of the objects.

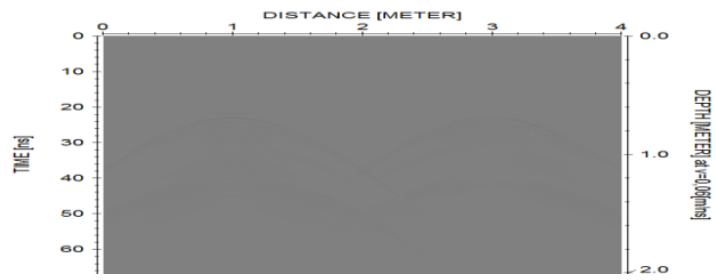

(a)

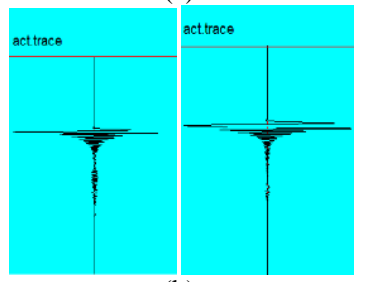

(b)

Fig.8. (a) Radargram of detection dielectric object placed above conductor object $\theta=0,45$; (b) evolution of the wave over time.
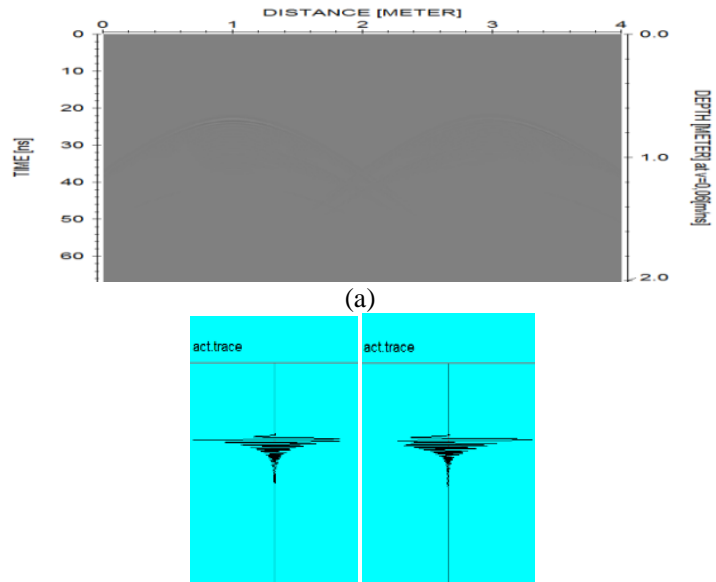

(b)

Fig.9. (a) Radargram of detection conductor object placed above dielectric object $\theta=0,45$;(b) evolution of the wave over time.

\section{Two conductor's objects placed perpendicular}

For the first situation we can note the interference between the reflected signals from objects (the objects on the left) (figure 10). While for the second situation the conductivity of the first object attenuates the wave and on the radargram we see only the hyperbola indicates the presence of the first object. 


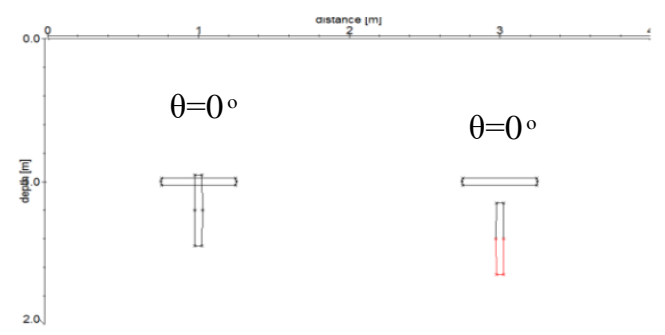

(a)

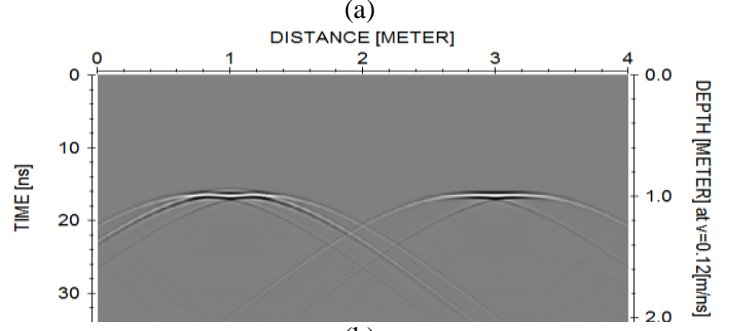

(b)

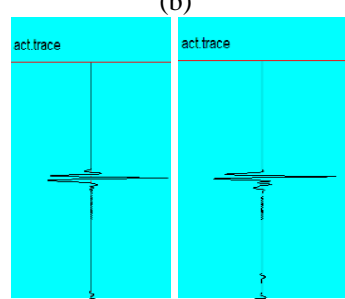

(c)

Fig.10. (a) Geometric two conductor objects placed perpendicular $\left(\theta^{\circ}=0^{\circ}\right)$ in a medium of dry sand;(b) radargram of detection objects and (c) evolution of the wave over time.

For the other case (figure 11) we can note the reflection from two objects (figure 11c) and the appearance of the hyperbolas indicate the existence of the two objects on the radargram.
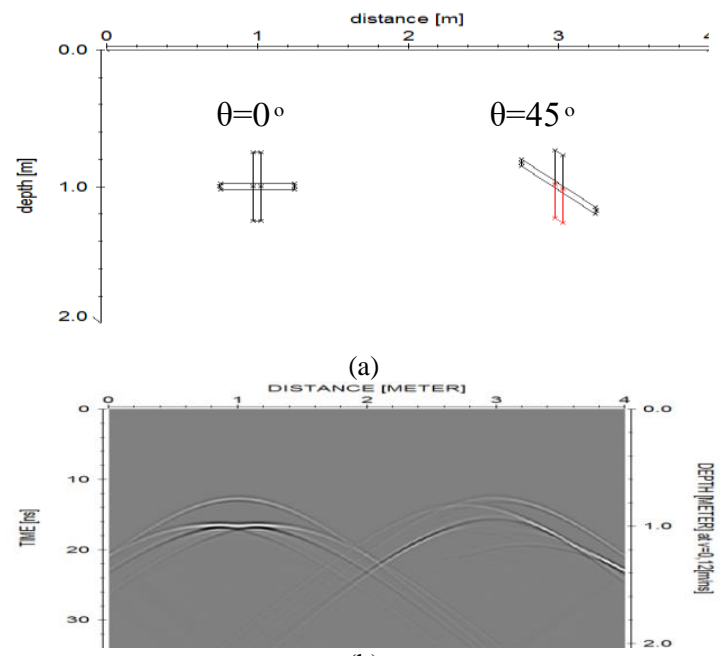

(b)

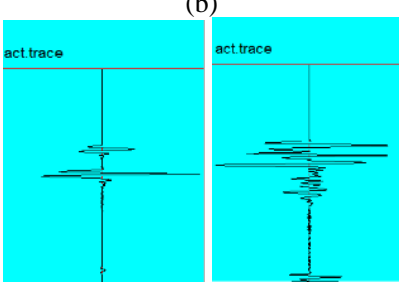

(c)

Fig.11. (a) Geometric two conductor objects placed perpendicular ( $\theta$ angel between antenna an object $=0$ and 45 )in a medium of dry sand;(b) radargram of detection objects and (c) evolution of the wave over time.

\section{E. Comparison between experimental and simulation result}

The aim of this section is to validate the simulation results obtaind using reflexw software by comparing them with the experimental result [11] under the same conditions. We note a good agreement between experimental and simulation result.

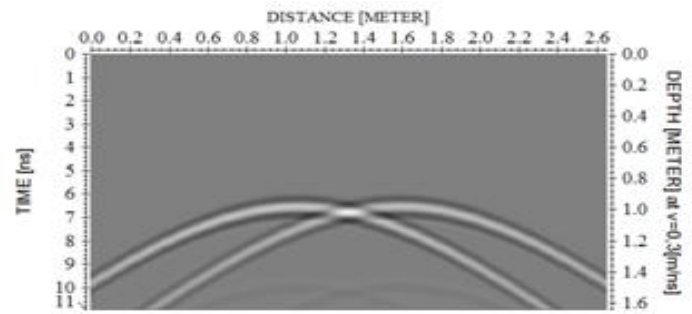

(a)

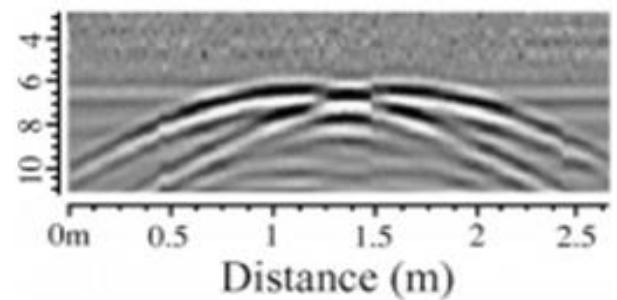

(b)

Fig.12.Radargramsdetecttwo conductor objects Placed in air at a depth of $91 \mathrm{~cm}$ (a) simulation result using Reflexw and; (b) experimental result[11].

\section{CONCLUSION}

The results of this study show that the GPR waves are strongly influenced by the orientation of objects. Therefore, the propagation path, the reflexion strength and the waveform change with the change of object orientation. The GPR waves are also very sensitive to changes in dielectric permittivity and electrical conductivity of objects. Moreover, it should be noted that the rapidity of the attenuation of GPR signal depends primarily on the electrical conductivity of objects. It is also noticeable that in the medium of wet sand the attenuation is very fast and the penetration depth is decreased significantly.

\section{REFERENCES}

[1] V. Montiel-Zafr, "A novel method to remove GPR background noise based on the similarity of non-neighboring region",Journal of Applied Geophysic,2017.

[2] X. Núñez-Nieto,"Signal-to-Noise Ratio dependence on Ground Penetrating Radar antenna frequency in the field oflandmine and UXO detection", Measurement 73 (2015) 24-32.

[3] Fabio Tosti, An investigation into the railway ballast dielectric properties using different GPR antennas and frequency systems", NDT and E International (2017).

[4] Monica Di Prinzio, "Application of GPR to the monitoring of river embankments", Journal of Applied Geophysics 71 (2010) 53-61.

[5] Wenzhe Fa, "Simulation for ground penetrating radar (GPR) study of the subsurface structure of the Moon", Journal of Applied Geophysics 99 (2013) 98-108

[6] Jing Li1, "GPR Polarization Simulation with 3D HO FDTD, 1College of Geoexploration Science and Technology", Jilin University,2010.

[7] Rebecca Ludwig, "Electromagnetic Methods in Applied Geophysics", Version: January 2011.

[8] [8] G.Alsharahi, "2D FDTD Simulation to Study Response of GPR Signals in Homogeneous and Inhomogeneous Mediums", International Journal on Communications Antenna and Propagation (IRECAP), Vol. xx, n. x June 2010. 
[9] Arnaud louis , "Détection des zones humides dans le béton par Ground Penetrating Radar en présenced'un gradient d'humidité, thése , université de Liége",2010-2011.

[10] Gamil Alsharahi, AMM .Mostapha, Abdellah Driouach and Ahmed Faize,"Modelling and Simulation Resolution of Ground-Penetrating Radar Antennas". Journal of electromagnetic engineering and science Volume 16, Issue 3, 2016, pp.182-190.

[11] F. I. Rial, M. Pereira,"Resolution ofGPR bowtie antennas: An experimental approach", Journal of Applied Geophysics, 67 (2009) 367-373.

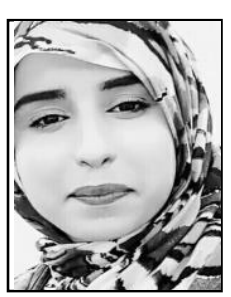

Aye Mint Mohamed Mostapha was born in Magtalahjar City, Mauritania in 1991. She got a professional license in applied physics option Electronic-Electro technical-Automatic and a master's degree in Telecommunication Systems Engineering from Abdelmalek Essaadi University, Faculty of Sciences, Tetouan. She is currently working toward a Ph.D degree at the same university.

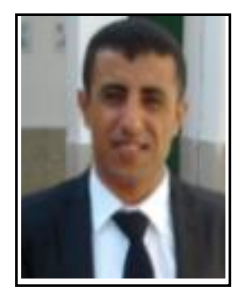

Gamil Alsharahi was born in Amran City, Yemen, in 1979. He received a B.S. in mathematics and physics and master's degrees in telecommunication and electronics from Abdelmalek Essaadi University, Faculty of Sciences, Tetouan. Currently, he is working toward a Ph.D degree at the same university.

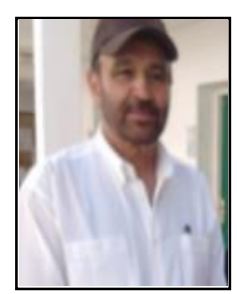

Abdellah Driouach was born in Al Hoceima City, Morocco, in 1954. He got his license in electronic physics at the University of Rabat (Morocco) in 1979, his doctorate (of third cycle) in "spectronomiehertzienne", at the University of Bordeaux 1 (France) in 1983, and his doctor title (insciences) at the University of Grenade (Spain). He has been a professor and researcher in the Faculty of Science, Abdelmallek Essaadi University since 1983. $\mathrm{He}$ has participated in several scientific research studies, including the dispersion of electromagnetic waves on obstacles to arbitrary structures. Currently, he is a member of the research team of communication systems. 